\title{
Une incursion dans les « coulisses » de la fabrication de la ville
}

The Making of the City: Behind the scenes

\section{Véronique Biau}

\section{(2) OpenEdition}

\section{Journals}

Édition électronique

URL : http://journals.openedition.org/crau/550

DOI : $10.4000 /$ crau. 550

ISSN : 2547-5746

Éditeur

Éditions du patrimoine

Édition imprimée

Date de publication : 1 novembre 2012

Pagination : 88-96

ISBN : 978-2-7577-0108-9

ISSN : 1296-4077

Référence électronique

Véronique Biau, «Une incursion dans les «coulisses » de la fabrication de la ville », Les Cahiers de la recherche architecturale et urbaine [En ligne], 26/27 | 2012, mis en ligne le 01 novembre 2017, consulté le 21 avril 2019. URL : http://journals.openedition.org/crau/550 ; DOI : 10.4000/crau.550 
Les " coulisses » de la fabrication de la ville sont l'objet d'analyse d'un certain nombre de chercheurs qui, travaillent, en équipes ou en réseaux, sur les processus et les enjeux qui sous-tendent aujourd'hui les pratiques de conception architecturale et urbaine en France et dans le monde occidental. Les évolutions en cours sont nombreuses et déterminantes. Cet article propose d'en faire un état.

\section{Une incursion dans les "coulisses" de la fabrication de la ville}

VÉRONIQUE BIAU

Si l'architecture et la ville viennent principalement à l'observateur par le biais des événements que constituent la livraison d'un édifice remarquable, la discussion autour des options liées à la conception d'espaces urbains, ou encore les difficultés économiques ou sociales que rencontrent tel ou tel quartier, toutes ces scènes prennent place par rapport à des " coulisses" où opère un système complexe de savoirs, savoir-faire, intentions, attentes, contraintes qui donnent corps et sens au projet architectural et urbain et aux politiques s'appliquant à la gestion des espaces habités.

Ces " coulisses " sont l'objet d'analyse d'un certain nombre de chercheurs qui, dans les laboratoires des écoles d'architecture, les instituts et départements d'urbanisme, de géographie ou de sociologie ou encore dans les grandes écoles travaillent, en équipes ou en réseaux 1 , sur les processus et les enjeux qui sous-tendent aujourd'hui les pratiques de conception architecturale et urbaine en France et dans le monde occidental.

1. On pense ici tout spécialement au réseau Ramau, fondé en 1998 habilité comme réseau de la recherche architecturale par la Direction générale des patrimoines (ministère de la

Culture et de la Communication).

On peut consulter le site

du réseau à l'adresse suivante

http://www.ramau.archi.fr 
On remarque que, sur les cinquante ou soixante ans d'existence en France d'une recherche architecturale instituée, et sur une toile de fond qui, dans nos domaines, donne une primeur à la matérialité des objets produits, ce type de travaux, qui articule la production bâtie et les ressorts économiques et sociaux qui forgent le contexte dans lequel elle opère, a pesé d'un poids fluctuant dans les débats. Ces dix dernières années ont été une période d'intense évolution des contextes et des pratiques. Pour s'en convaincre, il suffit de rapprocher les évolutions institutionnelles et économiques qui ont accompagné la réalisation de l'Union européenne et leurs impacts réglementaires sur les marchés et les professions, la montée des questions environnementales qui renouvelle le cadre épistémologique et éthique de l'action sur la ville (la longue durée, le rapport aux énergies, l'interdépendance des échelles), les essais en faveur d'une gouvernance urbaine plus partagée (démocratie participative, processus coopératifs), l'évolution même des structures, des compétences et des logiques de la maîtrise d'ouvrage et, en miroir, non seulement le questionnement identitaire mais aussi l'évolution organisationnelle profonde de divers profils professionnels agissant dans la conception, la programmation... Sans même évoquer le sort nouveau fait aux notions de qualité, de performance, de service, de partenariat public-privé, etc.

$C^{\prime}$ est sur ces divers points que ce texte va tenter de dessiner un état des lieux, en s'appuyant sur les travaux récents et en cours. Pour clarifier le propos, on peut scinder I'approche en trois cercles concentriques autour de l'activité de projet, du plus englobant au plus spécifique : un premier niveau d'orientation de l'action, qui se situe à l'échelle macro-sociale et intervient essentiellement dans la programmation et dans les procédures s'appliquant au projet architectural et urbain; un deuxième niveau qui se situe à l'articulation du concepteur et de la demande et/ou de la commande qui lui est adressée; et enfin un troisième niveau qui met en scène l'équipe de projet, avec ses compétences et ses structures hybrides, ses outils et la négociation d'objectifs partagés.

\section{Cadre, orientation de l'action}

Le «fond de scène ", ce cadre qui oriente les processus et les finalités de la conception architecturale et urbaine, relève de tendances lourdes. On ne l'évoquera que pour mémoire puisque dans ses grandes lignes il est connu de tous et fait l'objet de travaux nombreux et déjà anciens pour la plupart.

\section{Évolution des sociétés urbaines}

C'est tout d'abord l'évolution socio-démographique qui, à travers l'allongement de la vie, l'éclatement et les recompositions de la famille, les processus migratoires, pèse d'un poids considérable sur les parcs immobiliers et les dynamiques urbaines. L'évolution des modes de vie, avec un écart croissant entre les niveaux de vie des ménages, l'évolution de la nature et du statut du travail dans la vie quotidienne, l'évolution des mobilités entre autres interviennent pour leur part dans les solvabilités, les représentations du quartier, les réflexions sur l'organisation des agglomérations urbaines.

\section{Nouvelles logiques managériales}

Le tournant du millénaire a par ailleurs vu se dessiner des évolutions politico-institutionnelles et macro-éco- 
nomiques majeures, en prise avec le secteur du bâtiment d'une part, avec l'action publique locale d'autre part. C'est, en France, la poursuite de la décentralisation avec le rôle prépondérant, diversifié, contrasté, conflictuel, des collectivités locales sur les politiques urbaines (Bourdin-Prost, 2009). On continue à observer des formes de valorisation du « local » : après les grands projets de ville et le marketing urbain des années 1980, qui ont d'ailleurs accompagné l'émergence de réelles cultures techniques locales (en matière de projet urbain, d'équipements publics, de tramways, etc.), on observe maintenant une entrée des collectivités locales dans le knowledge management avec l'instauration de nouvelles coopérations entre universités, collectivités publiques et milieux professionnels, par exemple au sein de centres de ressources régionaux (Annales de la recherche urbaine, 2008).

Avec le retrait de l'État et le désengagement des pouvoirs publics dans les financements des équipements et services publics, et en ce domaine la France s'engage sur un chemin déjà bien connu de nombreux pays d'Europe du Nord, commencent à poindre dans la ligne du New Public Management des réflexions nouvelles sur la patrimonialité publique, fort éloignées de la tradition française en faveur d'une commande architecturale monumentale et emblématique. Par carence d'investissements publics à verser aux opérations urbaines et aux équipements publics, se développent des formes alternatives de production et de gestion : cession de bâtiments et terrains publics (en France, c'est la nouvelle politique immobilière de l'État, NPIE), transfert des budgets et des fonctions de gestion des équipements publics à des agences para-publiques jugées sur leur performance, recherche de bénéfices commerciaux au sein des bâtiments publics (politique britannique des Wider Markets), partenariats public-privé et diverses formes de privatisation... La mondialisation, par l'internationalisation de nombre d'acteurs de la conception et de la construction ainsi que d'opérateurs de services urbains, favorise le développement de «bonnes pratiques " tendant à unifier tant les processus que les formes bâties (Espaces et sociétés, 2007). II en est de même avec la mise en place de l'Union européenne qui a deux types d'impact : elle a bien sûr des impacts directs, par la surimposition d'un cadre juridique et réglementaire européen relevant parfois d'autres logiques que le droit national français, en matière de professions ou de marchés publics par exemple ("directive architecture ", " directive services ») avec la montée de l'impératif de "libre-concurrence », la tendance à la dérégulation, etc. ; mais on peut aussi lui voir des impacts indirects par exemple avec la mise en débat de questions d'organisation, de marchés, d'identités professionnelles, jusquelà peu prisées par le milieu ou encore par la circulation transfrontalière accrue de pratiques et de controverses concernant la conception, le projet mais aussi l'environnement professionnel de cette activité.

\section{De la " crise urbaine" aux valeurs consensuelles}

Sur le plan des valeurs et des doctrines qui sous-tendent I'action architecturale et urbaine, les travaux montrent un glissement progressif, plus sensible au cours des deux dernières décennies, d'un diagnostic de "crise », 
accompagné par de nombreux blocages dans la production urbaine (infrastructures, ZAC, permis de construire) vers l'émergence de nouvelles valeurs consensuelles (BiauTapie, 2009). Ainsi un certain nombre de valeurs humanistes et patrimoniales se sont imposées, qui transcendent les débats politiques et jouent un certain rôle de pacification par rapport aux craintes et aux hostilités engendrées par une urbanisation consommatrice d'espace et d'énergie, et révélatrice de tensions sociales.

La démocratie participative se situe dans ce registre, visant l'adhésion du plus grand nombre aux décisions urbaines, dans un contexte marqué par la crise de la représentation politique. On y retrouve bien sûr, sous une forme plus pragmatique, les mouvements de luttes urbaines et d'advocacy planning des années 1970. Mais l'objectif est maintenant d'instaurer des relations permanentes, directes, et contrôlées avec le plus grand nombre d'habitants possible avant de déterminer les actions à entreprendre, manière de désamorcer les conflits potentiels. Information des habitants, concertation, co-conception, la forme des relations diffère d'une situation à une autre; le cadre des négociations aussi : référendums, conseils de quartier, budgets participatifs, etc. (Bacqué-Rey-Sintomer, 2010). Ce qui en résulte, pour les architectes, comme pour les autres acteurs de la réflexion urbaine, est un déplacement des attentes à leur égard : il s'agit moins de formuler une solution à un programme dans un système de contraintes donné, que de participer à la construction collective des problèmes liés à l'espace.

Le thème du développement durable $a$, plus encore que celui de la démocratie participative auquel il est lié, un effet consensuel. Face à une menace planétaire, s'imposent à tous niveaux les discours de préservation des ressources et des équilibres. Les traductions de ces mots d'ordre diffèrent fortement d'un pays à un autre mais le projet s'inscrit désormais dans une conscience plus aiguë de l'interdépendance des échelles (Tsiomis, 2007), du micro-local au planétaire. Et au fur et à mesure que s'affinent les politiques et les pratiques environnementales, se pose la question : I'introduction de ce facteur dans la pensée architecturale et urbaine va-t-elle se traduire principalement par un nouveau corps de normes venant se juxtaposer aux normes thermiques et constructives existantes ou va-t-elle engendrer une véritable "rupture paradigmatique ", une refonte globale des modes de travail du secteur prenant davantage en considération le temps de la gestion des bâtiments, les ressources incorporées dans les matériaux, la question de la démolition et des déchets, etc. ? (Cooper, Symes, 2009).

\section{Le concepteur et l'évolution de la commande qui lui est adressée}

L'activité de conception se développe dans un contexte de commande qui, lui aussi, connaît des évolutions rapides, et que la recherche des dix dernières années a tenté de mettre en lumière. Un pan de cette évolution tient à la nature des acteurs en charge de la formulation de cette commande; l'autre pan en résulte : la conduite des processus, les attentes à l'égard des maîtres d'œuvre s'infléchissent et avec elles le cadre opératoire des professionnels de la conception. 


\section{Des clients hybrides}

Tout d'abord, c'est la complexification de la commande qui est notée : la fonction de client relève de plus en plus de collectifs qui rassemblent des logiques diverses voire antagonistes et font intervenir des arbitrages internes (Bonnet-Claude, 2001). Dans le secteur public, il est fréquent que les opérations relèvent d'une multiplicité d'échelles territoriales (État, région, département, ville, intercommunalité et éventuellement Europe) participant au financement des opérations; cela engendre controverses et incertitudes sur le " portage » et sur le leadership politique et technique des projets et des opérations. Dans le secteur privé, les maîtres d'ouvrage relèvent de firmes de plus en plus importantes, internationales et organisées, du fait de la concentration horizontale et verticale du secteur BTP engagée depuis les années 1980, ainsi que de la mondialisation des opérateurs immobiliers. La décision immobilière, qui s'exerce au sein de commissions, de comités de pilotage ou de groupes de projet, relève alors de plusieurs directions (direction technique, direction du patrimoine, direction de I'investissement ou leurs équivalents) dans lesquels profils professionnels et logiques d'action diffèrent. La notion de «forum hybride » (Callon-Lascoumes-Barthes, 2001), a d'ailleurs été largement mobilisée par les chercheurs sur la ville, montrant comment des dispositifs associant la puissance publique, les acteurs privés (investisseurs, promoteurs), les associations de riverains, etc. avaient été constitués autour d'opérations complexes et lourdes d'enjeux, impliquant des négociations et des arbitrages plus explicites sur la diversité des attentes autour du projet urbain et architectural.

\section{Une régression constante de la part de commande publique}

Par ailleurs, se confirme la montée de la commande privée par rapport à la commande publique, à laquelle le champ de l'architecture français continue, selon une longue tradition, à accorder une place prépondérante dans la définition des personnalités, des modèles et des discours qui structurent le débat architectural. La commande publique, qui représentait $44,8 \%$ des revenus globaux des architectes français en 1983, a structurellement décru jusqu'à $30 \%$ en 2010 (chiffres CNOA). Or, on le sait, les modalités de dévolution des marchés, les relations de travail mais aussi les horizons d'attente, les attitudes culturelles des commanditaires envers l'architecture, diffèrent largement dans le secteur public et dans le secteur privé. Pour les architectes, ce sont aussi des stratégies différentes d'accès aux marchés, des prestations différentes, des bénéfices différents en termes de reconnaissance symbolique, qui tendent à une fermeture des milieux professionnels respectifs autour des diverses formes de "clientélisme " qui y ont cours.

\section{Une maîtrise d'ouvrage professionnalisée}

Dans les deux secteurs, public et privé, la maîtrise d'ouvrage connaît une professionnalisation et une sophistication substantielles. Des formations de haut niveau se sont diffusées qui ont porté aux postes de direction des agents spécialisés, très qualifiés, et orientés par la recherche d'un meilleur contrôle des processus : en matière $d$ 'ingénierie financière et de gestion des coûts, on voit s'affiner la connaissance de l'économie du projet; ratios, grilles de prix, benchmarking, calcul 
en coût global, etc. (Terrin, 2005). La gestion des risques, dans une acception large du terme qui s'ouvre bien au-delà des risques techniques liés à la construction englobe désormais tous les aléas dont on peut anticiper la survenue sur la longueur du cycle de vie du bâtiment : risques administratifs liés aux changements réglementaires, risques naturels, risques liés à l'exploitation (évolution des publics, des besoins). Parallèlement, la gestion de la qualité s'introduit dans les milieux du bâtiment en provenance des mondes industriels, que cela soit sur le contrôle du procès de production sur le chantier ou sur la prestation de service dévolue au concepteur: elle diffuse l'idée d'une amélioration des pratiques par une capitalisation plus systématique des résultats de l'expérience et une importance plus grande accordée à l'évaluation. À cette sophistication des compétences de la maîtrise d'ouvrage dans une part non négligeable de ses structures correspond l'élévation du niveau de ses exigences à l'égard des architectes : explicitement ou non, il devient important dans les marchés concurrentiels sur lesquels ils se situent qu'ils puissent témoigner d'une certaine connaissance de ces questions, qu'ils acceptent de participer à des protocoles de gestion et de suivi, qu'ils rendent " traçables » les étapes du projet et leur validation par exemple.

\section{La prégnance des processus rationalisés anglo-saxons}

Les analyses comparatives internationales, rendues plus nombreuses par les processus de globalisation et les besoins de connaissances qui en découlent, montrent bien l'empreinte des modes de pensées du monde anglo-américain et scandinave sur la gestion du projet architectural et urbain. La diffusion à l'échelle mondiale des contrats globaux de type " conception-construction » ou " partenariats public-privé » sur le modèle des Private Finance Initiatives britanniques en est une claire illustration. Des dispositifs anglo-saxons nous arrivent en effet $^{2}$ qui sont autant de recompositions des partenariats engagés dans le projet. Outre la complexité de la mise en place de ces procédures, les architectes font l'expérience, dans ces diverses situations, d'un " glissement culturel » dans la définition de leur mode d'intervention. Tout d'abord, ces contrats globaux sont le reflet et le vecteur d'une primauté accordée aux logiques techniques d'une part, aux mécanismes juridico-financiers d'autre part, dans le déroulement du processus de montage et de conception du projet (Campagnac, 2009).

La prestation de l'architecte et des autres maîtres d'œuvre le cas échéant est alors fortement encadrée par les groupes bancaires, les entreprises de construction, les sociétés de service en charge de l'exploitation du bâtiment, séparément ou groupés au sein d'un consortium dont la tendance est de faire de la conception une mission sous-traitée. L'une des répercussions, et pas des moindres, peut être la disparition d'une interlocution directe entre le concepteur et son client-utilisateur. La conception est évaluée essentiellement sur des critères de performance organisationnelle et économique et cela surtout dans les pays où la notion de Best value for money s'est imposée fortement, comme en Grande-Bretagne. Les contrats de partenariat français, tout comme les Private

2. Le DB (Design and Build), le DBO (Design, Build and Operate), le DBFO (Design, Build, Finance and Operate) 
Finance Initiatives britanniques qui sont des contrats DBFO selon la typologie évoquée, sont très illustra-

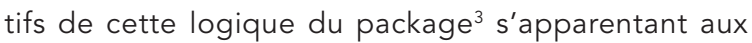
formules "clés en main ": la commande n'est alors plus celle d'un bâtiment, dans ses caractéristiques urbaines, volumétriques, plastiques voire esthétiques mais celle d'une enveloppe matérielle optimisant l'ensemble des flux et des prestations requises par l'organisation du service qu'il est supposé rendre (Carassus, 2003). En d'autres termes, on voit une nette translation d'une logique de l'objet, dans sa matérialité et sa spécificité, vers une logique de service engendrant un déplacement du centre de gravité depuis les questions liées à la production vers celles de la gestion. Cette évolution en germe est particulièrement déconcertante dans le contexte de la commande publique française qui, depuis des siècles, associe étroitement l'expression du pouvoir politique, la monumentalité et/ou l'originalité du bâtiment, et la définition des élites architecturales.

\section{L'équipe de projet, négociation d'objectifs partagés Du " séquentiel " au " concourant ", la diffusion de la tâche de conception}

Depuis une quinzaine d'années, les travaux sur l'ingénierie et la gestion de projet ont mis en lumière une réorganisation de la tâche de conception et un glissement de la place de l'architecte dans I'organisation globale du processus de production des opérations. L'architecte s'est longtemps représenté et a longtemps été reconnu comme "chef d'orchestre » d'un processus séquentiel reposant sur une chaîne d'acteurs ayant chacun une compétence partielle sur un moment du processus. Mais la complexité croissante des opérations, la spécialisation au sein de la conception (concepteurs de façade, spécialistes en énergies renouvelables, concepteurs-lumière...), la compétence accrue en matière de conception d'autres acteurs de la chaîne (programmation, conception technique, conception de la maintenance), l'avènement des technologies de I'information qui facilitent les échanges et les conceptions en parallèle remettent en cause cet état de fait. Même dans des processus non explicitement coopératifs, les partenariats s'accentuent, les inputs sur la conception dans des termes aussi divers que l'économie, la gestion, la durabilité environnementale, les choix techniques, les usages se multiplient en provenance d'acteurs et partenaires multiples (Ben Mahmoud-Jouini, 2003). Aux États-Unis, la question se pose d'ailleurs de définir, dans leurs répercussions juridiques, les parts de responsabilité prises par les divers acteurs dans les tâches de conception (Bernstein-Deamer, 2010). En tout état de cause, toutes les formes de coordination et de négociation, les missions de pilotages prennent de l'importance et mettent en avant d'une manière accentuée les capacités relationnelles des acteurs (Évette-Terrin, 2006).

\section{Réflexion sur l'organisation et l'évaluation de la prestation architecturale}

Catalysée par les confrontations avec leurs semblables européens, une réflexion se développe chez les architectes français sur l'organisation des agences, l'organisation du travail, la nature de la prestation
3. Nous nous permettons ici cet anglicisme parce qu'il permet d'évoquer la diversité des prestations négociées et traitées dans un contrat unique, introduisant des rapports de force plus directs et plus explicites entre les acteurs financiers et techniques et les concepteurs. Ce terme est aussi, avec celui de bundled, utilisé en Grande-Bretagne pour désigner ceux de ces contrats qui, par souci d'économies d'échelles, peuvent englober sous un contrat unique le financement, la conception, la réalisation et l'exploitation pendant plus de 20 ans de plusieurs unités ou de plusieurs dizaines d'équipements (tribunaux, commissariats, dispensaires, écoles...). 
qu'ils tendent à offrir (Tapie, 2000; Chadoin, 2007). L'accès aux commandes importantes et aux marchés à l'exportation exige de plus en plus une agence pluridisciplinaire, dotée de références et de moyens concurrentiels par rapport à leurs confrères britanniques ou néerlandais, par exemple. L'exercice en sociétés, les fusions, la mise en commun de moyens sont dans I'air du temps, amenant les architectes à accorder davantage d'intérêt à l'efficacité du travail dans l'agence. Le décompte des heures, l'adoption d'une comptabilité analytique, la rationalisation d'un certain nombre de tâches subalternes en sont autant d'illustrations. Il apparaît aussi que parallèlement à certains maîtres d'ouvrage, les architectes se penchent sur les démarches d'organisation de la qualité en s'inscrivant dans les protocoles ou seulement en s'inspirant des principes des procédures de type Iso. Dans cette mouvance, une attention se développe sur les formes de capitalisation de l'expérience partant de l'évaluation et de la correction continue de l'action par les retours que peuvent en donner partenaires et clients, audits internes et externes. De même, par rapport au cadre technico-économique de leur action, toujours plus prégnant comme on l'a vu, se développent des postures "stratégiques » chez les architectes: il s'agit moins qu'auparavant de nier ce type de contraintes ou d'essayer de "passer en force " par rapport à elles, mais plutôt de tenter de les connaître au mieux, de les devancer dans les négociations, de hiérarchiser I'impact que le projet veut leur accorder, pour maintenir un certain souci de qualité, sous les critères qui priment pour le concepteur (Biau-Lautier, 2009; Hoddé, 2006).

\section{En guise de conclusion}

Parce qu'ils croisent des observations et des analyses qui se portent en divers lieux du champ professionnel, pas nécessairement appréhendables dans leur ensemble par les praticiens, les travaux de recherche s'intéressant aux « coulisses » de la fabrication de la ville font surgir un certain nombre de questions d'importance par rapport aux jeux d'acteurs qui s'y déroulent. On en pointera ici trois familles. D'une part, la rigidification des cadres procéduraux et des contraintes économiques, se doublant d'une concurrence accrue des acteurs et opérateurs à l'échelle nationale et internationale, renversent l'ordre des valeurs engagées dans le projet, tant architectural qu'urbain : la dimension esthétique et signifiante de l'architecture et de l'aménagement urbain ne disparaît pas mais entre dans des itérations plus serrées avec la gestion des coûts, des délais, des risques, ainsi bien sûr qu'avec l'évaluation de leurs impacts environnementaux. Liée à ces repositionnements, une deuxième famille de questionnements se focalise sur les identités professionnelles, les compétences, les savoir-faire; ces réflexions ne semblent épargner aucun des groupes professionnels intervenant dans la production urbaine et transparaissent sur les formations, les débats propres aux organisations professionnelles, etc. Enfin, comme dans de nombreux secteurs d'activité, une logique inéluctable d'approfondissement des savoirs, de spécialisation et de segmentation des missions marque le secteur de la fabrication de la ville et l'on posera ici l'hypothèse, pour l'observation des transformations en cours, que l'espace sera l'œuvre de ceux de ces acteurs qui parviendront à s'imposer et 
se faire accepter comme spécialistes de la synthèse, aptes à conduire les processus sur la longue durée, de l'émergence de l'idée aux temps longs de la gestion des espaces, et à assumer ce " défi intellectuel de la chaîne intégrée " (selon l'expression des Anglo-Saxons) qui est celui des prochaines décennies.

- Annales de la recherche urbaine $n^{\circ} 104$, «L'expertise au miroir de la recherche ", juillet 2008.

- Marie-Hélène Bacqué, Henri Rey, Yves Sintomer (dir.), Gestion de proximité et démocratie participative. Une perspective comparative, Paris, La Découverte, 2005.

- Sihem Ben Mahmoud-Jouini, Co-conception et savoirs d'interaction, Paris, PUCA, 2003.

- Phillip Bernstein, Peggy Deamer (dir.), Building (in) the Future : Recasting Labor in Architecture, New York, Princeton Architectural Press, 2010.

- Véronique Biau, Guy Tapie (dir.), La fabrication de la ville; métiers et organisations, Marseille, Éditions Parenthèses, coll. " La ville en train de se faire ", 2009.

- V. Biau, François Lautier (dir.), " Acteurs et enjeux de la qualité architecturale ", Cahiers Ramau n ${ }^{\circ}$, Paris, Éditions de la Villette, 2009.

- Michel Bonnet, Viviane Claude, Michel Rubinstein (dir.), La commande... De I'architecture à la ville, Paris, Éditions du PUCA, coll. « Recherche " n 138, 2001.
- Alain Bourdin, Robert Prost, Projets et stratégies urbaines. Regards comparatifs, Marseille, Éditions Parenthèses, 2009.

- Michel Callon, Pierre Lascoumes, Yannick Barthes, Agir dans un mode incertain. Essai sur la démocratie technique, Paris, Le Seuil, coll. " La couleur des idées ", 2001.

- Élisabeth Campagnac (dir.), Évaluer les partenariats public-privé en Europe. Quelles conséquences sur la commande et le projet? Quels impacts sur la qualité des bâtiments et des services? Quelles méthodes d'évaluation?, Paris, Presses de I'École nationale des Ponts et Chaussées (ENPC), 2009.

- Jean Carassus, Construction : la mutation. De l'ouvrage au service, Paris, Presses de l'École nationale des Ponts et Chaussées (ENPC), 2003.

- Olivier Chadoin, Être architecte : les vertus de l'indétermination. De la sociologie d'une profession à la sociologie du travail professionnel, Limoges, Éditions Pulim, 2007.

- Ian Cooper, Martin Symes et al.,
Sustainable Urban Development, vol. 4, Changing professional Practice, New York, Routledge, 2009.

- Espaces et sociétés n 131, «Villes et "best practices" ", Paris, Éditions Érès, décembre 2007.

- Thérèse Évette, Jean-Jacques Terrin (dir.), « Expertises, concertation et conceptions. Projets urbains ", Cahiers Ramau $n^{\circ} 4$, Paris, Éditions de la Villette, 2006.

- Rainier Hoddé, Qualités architecturales : conceptions, significations, positions, Paris, Éditions Jean-Michel Place, 2006.

- Guy Tapie, Les architectes : mutations d'une profession, Paris, L'Harmattan, 2000.

- J.-J. Terrin (dir.), Maîtres d'ouvrage, maîtres d'œuvre et entreprises. De nouveaux enjeux pour les pratiques de projet, Paris, Eyrolles-pucA, 2005.

- Yannis Tsiomis, Échelles et temporalités des projets urbains, Paris, Éditions Jean-Michel Place, 2007. 